\title{
ACOLHIMENTO COM CLASSIFICAÇÃO DE RISCO: UM INDICADOR DA DEMANDA EMERGENCIAL DE UM SERVIÇO HOSPITALAR*
}

Thaise Honorato de Souza', Selma Regina de Andrade²

'Enfermeira. Mestranda do Hospital Universitário da Universidade Federal de Santa Catarina. São José-SC-Brasil. 2Enfermeira. Doutora em Enfermagem. Universidade Federal de Santa Catarina. Florianópolis-SC-Brasil.

RESUMO: Este estudo objetivou caracterizar e analisar a porta de entrada e possíveis estratégias para organização do Serviço de Emergência de um Hospital Universitário, considerando o acolhimento com classificação de risco, a Rede de Atenção às Urgências e a Rede de Atenção à Saúde do Sistema Único de Saúde. Estudo descritivo, transversal, realizado entre nove de abril e oito de junho de 2012. Obteve-se um total de 3.078 casos de acolhimento. Os dados foram coletados por meio de observação participante e de consulta documental, analisados por temática e estatística descritiva. Os resultados mostraram que $63,55 \%$ dos acolhidos acessaram o referido serviço devido a casos não emergenciais. Após caracterização, discutiram-se alguns elementos para organização da demanda emergencial na perspectiva de rede de atenção. O estudo mostrou que apesar da classificação de risco ser uma estratégia inovadora, por si só não soluciona os problemas de excesso de demanda não emergencial. DESCRITORES: Enfermagem; Serviço hospitalar de emergência; Serviços de saúde; Sistema Único de Saúde.

\section{EMBRACEMENT WITH RISK CLASSIFICATION: AN INDICATOR OF THE EMERGENCY DEMAND ON A HOSPITAL SERVICE}

\begin{abstract}
This study aimed to characterize and analyze the gateway and possible strategies for organization of the Emergency Service of a University Hospital, considering the embracement with classification of risk, the Emergency Care Network and the Healthcare Network of the Unified Health System. This descriptive, transversal study was undertaken between 9th April and 8th June 2012. A total of 3078 cases of embracement was obtained. The data were collected through participant observation and documental consultation, and were analyzed by thematic and descriptive statistics. The results showed that $63.55 \%$ of those embraced access the above-mentioned service due to non-emergency cases. After characterization, some elements were discussed for the organization of the emergency demand regarding the care network. The study showed that in spite of the risk classification being an innovative strategy, on its own it does not resolve the problems of excess nonemergency demand.
\end{abstract}

DESCRIPTORS: Nursing; Hospital emergency service; Health services; Unified Health System.

\section{ACOGIDA CON CLASIFICACIÓN DE RIESGO: UN INDICADOR DE LA DEMANDA EMERGEN- CIAL DE UN SERVICIO HOSPITALAR}

RESUMEN: Este estudio tuvo el objetivo de caracterizar y analizar la puerta de entrada y posibles estrategias para organización del Servicio de Emergencia de un Hospital Universitario, considerando la acogida con clasificacióno de riesgo, la Red de Atención a las Urgencias y la Red de Atención a la Salud del Sistema Único de Salud. Estudio descriptivo, transversal, realizado entre nueve de abril y ocho de junio de 2012. Se obtuvo un total de 3.078 casos de acogida. Los datos fueron recogidos por medio de observación participante e de consulta documental, analizados por temática y estadística descriptiva. Los resultados apuntaron que $63,55 \%$ de los acogidos accessaron el referido servicio a causa de casos no emergenciales. Después de la caracterización, fueron discutidos algunos elementos para organización de la demanda emergencial en la perspectiva de red de atención. El estudio mostró que a pesar de la clasificación de riesgo ser una estrategia innovadora, sola no resuelve los problemas de exceso de demanda no emergencial.

DESCRIPTORES: Enfermería; Servicio hospitalar de emergencia; Servicios de salud; Sistema Único de Salud.

*Estudo resultante do Trabalho de Conclusão da Residência Integrada Multiprofissional em Saúde. Hospital Universitário da Universidade Federal de Santa Catarina, 2012.

Autor Correspondente:

Thaise Honorato de Souza

Universidade Federal de Santa Catarina

Rua José Bernardo da Silva, nº149 - 88106-070 - São José-SC-Brasil

E-mail: thaisetise@yahoo.com.br
Recebido: 01/04/2013 Finalizado: 01/09/2014 


\section{INTRODUÇÃO}

Dentre os avanços que vêm materializandose na organização do Sistema Único de Saúde (SUS) está a implantação da Rede de Atenção à Saúde (RAS). No campo emergencial, há um conjunto de unidades prestadoras de serviços de saúde atuando e funcionando como porta de entrada para o sistema de saúde, isto é, prestando o primeiro atendimento ao usuário do $S U S^{(1)}$. Tais unidades compõem a Rede de Atenção às Urgências (RUE) ${ }^{(2)}$ e incluem os serviços de atenção primária; atenção de urgência e emergência; atenção psicossocial; e especiais de acesso aberto, por exemplo, centro de referência em AIDS, saúde do trabalhador e outros que atendam necessidades específicas do cidadão.

A RUE, apesar de ser um importante componente da assistência à saúde, constituise ainda uma das áreas problemáticas do SUS. Isso decorre de uma frágil estruturação da rede, que somada a um número cada vez maior de acidentes e violência urbana, resulta em uma sobrecarga de demanda no âmbito hospitalar ${ }^{(3)}$.

$\mathrm{O}$ aumento gradativo por atendimento nos serviços de emergência em hospitais privados e públicos leva, muitas vezes, a pessoa que necessita de um atendimento de emergência real, com risco iminente de morte, a aguardar por horas pela assistência. Isto decorre, em parte, pelo fato de que poucas pessoas que procuram estes recursos apresentam problemas de saúde efetivamente de caráter prioritário de urgência ou emergência, sendo que a maioria (65\%) dos casos poderia ser atendida em outro ponto de atenção da rede de saúde ${ }^{(4-7)}$. A situação nos serviços de emergência é agravada por problemas do tipo organizacional, como, por exemplo, a falta de triagem de risco, o que determina o atendimento por ordem de chegada, sem qualquer avaliação prévia do caso, resultando, muitas vezes, em agravos ainda maiores à saúde das pessoas ${ }^{(2,8)}$.

Dentre os modelos de triagem de risco, os mais avançados, de concepção sistêmica, utilizados por uma rede de serviços estão incluídos o Australiano, o Canadense, o Americano, o Andorra e o Sistema Manchester de Classificação de Risco ${ }^{(8)}$. O protocolo de Manchester de classificação de risco foi criado com o objetivo de estabelecer um consenso entre médicos e enfermeiros, quanto à ferramenta de classificação de risco, para os serviços de urgência e emergência. Tem como características principais uma escala em cinco níveis por cores, com base em categorias de sintomas, discriminantes-chave, algoritmos clínicos, e com um tempo de execução inferior a três minutos ${ }^{(8)}$.

No Brasil, as diretrizes gerais para a implementação da Política Nacional de Humanização ( $\mathrm{PNH}$ ) propõem, em âmbito hospitalar, o acolhimento com avaliação de risco, considerando este uma das ações potencialmente decisivas para a reorganização da atenção à saúde em rede ${ }^{(9)}$.

Ao adotar os pressupostos de que os serviços de emergência hospitalar (SEH) configuram um ponto de atenção relevante para a RAS e RUE, e que a implementação do acolhimento com classificação de risco é um elemento racionalizador da atenção às urgências, compreende-se que a identificação de fragilidades enfrentadas por um Serviço de Emergência Adulto (SEA) de um Hospital Universitário (HU) permitirá contribuir para melhor organização deste serviço. Com esta perspectiva, este estudo orienta-se pelas seguintes questões: Como se caracteriza a porta de entrada do SEA/HU? De que maneira é possível contribuir para melhor qualificar a porta de entrada hospitalar aos usuários do SUS?

Assim, considerando as diretrizes do acolhimento com classificação de risco e os elementos constituintes da RAS e da RUE, objetivase caracterizar e analisar a porta de entrada e possíveis estratégias para organização do SEA/HU.

\section{MÉTODO}

Estudo do tipo descritivo transversal, com a finalidade de caracterizar a demanda da porta de entrada em emergência hospitalar, junto ao SEA/ $\mathrm{HU}$ da cidade de Florianópolis, SC.

Os dados foram coletados por meio de consulta aos registros formais do primeiro contato com o usuário, atendido e classificado de acordo com seu grau de risco no SEA/HU, no período de nove de abril a oito de junho de 2012. Os dados utilizados no instrumento de coleta incluíram: local de procedência do usuário; procura por outro serviço de saúde antes de se dirigir ao SEA; queixa principal; o código do fluxograma utilizado de acordo com sua queixa principal e classificação de risco. Somente os registros dos 
pacientes com queixas clínicas foram incluídos no estudo, visto que os pacientes com demanda cirúrgica ou ginecológica não passam pelo processo de classificação de risco realizado pelo enfermeiro.

Foram realizados 3.249 registros de acolhimento no período estudado. Destes, 1.534 foram realizados nos primeiros 30 dias e 1.715 nos 30 dias seguintes. Contudo, da população de estudo foram excluídos 70 casos encaminhados à clínica cirúrgica; 05 encaminhados ao setor de ginecologia; e 96 que não realizaram a classificação, resultando um total de 3.078 casos participantes, dos quais 1.451 (de nove de abril a oito de maio, correspondendo ao Mês um) e 1.627 (de nove de maio a oito de junho, correspondendo ao Mês dois). Estes dados foram analisados mediante estatística descritiva, com apoio da planilha Excel®.

Complementarmente, durante o período do estudo, foram coletados dados por meio de observação participante pela pesquisadora responsável, em momento específico da realização da Residência Multiprofissional em Saúde. Com registros sistemáticos em diário de campo, observaram-se aspectos relativos ao primeiro atendimento (acolhimento) ao usuário, incluindo: circunstâncias dos atendimentos; comunicação entre os profissionais; situações de conflito; quantitativo de pessoal; demanda ao serviço; ocorrência de evento fortuito. Os registros da observação foram analisados segundo a técnica de análise temática ${ }^{(10)}$, seguindo as fases de ordenação dos dados, classificação em estruturas de relevância, síntese e interpretação dos dados, com a finalidade de contribuir para a proposição de estratégias de (re)organização do SEA/HU ${ }^{(11)}$.

Este estudo foi aprovado pelo Comitê de Ética em Pesquisa, sob parecer n 2438/2012, seguindo as recomendações da Resolução 196/96 do Conselho Nacional de Saúde.

\section{RESULTADOS}

O SEA/HU, visto como "centro nervoso" da instituição, presta atendimento de emergência 24 horas, em uma média de 400 pacientes/dia, que chegam por livre demanda ou referenciada de outras unidades ou serviços. Por se situar em uma capital em região litorânea, há uma grande variação no número de atendimento por mês, sendo este maior ou menor de acordo com a época do ano e/ou evento (veraneio/ inverno, período letivo/férias escolares, eventos científico-culturais, entre outros). O perfil dos pacientes assistidos no SEA/HU é caracterizado por idade acima de 14 anos, sendo a faixa etária de 45-60 anos a de maior prevalência. É, portanto, uma unidade que apresenta uma grande diversidade de faixa etária, compreendendo os mais diversos estados de gravidade das situações clínicas e cirúrgicas de saúde e todos os graus de dependência de cuidado de enfermagem.

O SEA/HU está dividido em três postos de atendimento: Acolhimento com classificação de risco, Serviço de emergência interna (SEI) e o Repouso da emergência. No acolhimento com classificação de risco são utilizados fluxogramas adaptados do protocolo de Manchester, com o critério de seis cores: vermelho (emergente), laranja (muito urgente), amarelo (urgente), verde (pouco urgente), azul (não urgente), branco (procedimento).

As características da porta de entrada do SEA/ $\mathrm{HU}$ foram identificadas com base nos registros dos acolhimentos realizados com fluxogramas adaptados do protocolo Manchester. No período de estudo, foram efetivamente classificados 3.078 casos, segundo o critério de cores (Tabela 1).

Tabela 1 - Distribuição de usuários classificados de acordo com protocolo de Manchester adaptado. SEA/ HU. Florianópolis-SC-Brasil, 2012

\begin{tabular}{lcccc}
\hline Classificação/Cor & Mês $\mathbf{1}$ & Mês $\mathbf{2}$ & Total & \% \\
\hline Vermelho & 03 & 03 & 06 & 0,19 \\
\hline Laranja & 41 & 64 & 105 & 3,41 \\
\hline Amarelo & 520 & 491 & 1011 & 32,85 \\
\hline Verde & 843 & 1012 & 1855 & 60,27 \\
\hline Azul & 14 & 41 & 55 & 1,79 \\
\hline Branco & 30 & 16 & 46 & 1,49 \\
\hline Total & 1451 & 1627 & 3078 & 100 \\
\hline
\end{tabular}


Os casos recebidos por encaminhamento interno (ambulatório) e por serviço de emergência pré-hospitalar não são registrados no acolhimento com classificação de risco. Assim, há uma possível margem de erro - para menos - nos dados aqui apresentados, com relação ao total de usuários atendidos no SEA/HU.

Os dados relativos à classificação de risco no SEA/HU mostram que $63,55 \%$ dos casos são não emergenciais, ou seja, casos solucionáveis em outro nível da RAS/RUE do SUS. Pacientes classificados com as cores laranja e vermelho, que representam casos graves, constituem uma minoria (3,6\%). Somados aos casos classificados na cor amarela, o total de condições emergentes, muito urgentes e urgentes representam pouco mais que a terça parte dos acolhimentos neste serviço.

Dentre as queixas registradas como "queixa principal" do usuário que procura o SEA/HU destacaram-se entre as cinco primeiras: dor abdominal, mal estar em adulto, cefaleia; dor lombar; e dor torácica, correspondendo a mais de $50 \%$ das queixas para atendimento (Tabela 02).

Tabela 2 - Distribuição da queixa principal dos usuários do SEA/HU. Florianópolis-SC-Brasil, 2012

\begin{tabular}{lcccc}
\hline $\begin{array}{l}\text { Protocolo adaptado } \\
\text { fluxograma utilizado }\end{array}$ & $\begin{array}{c}\mathbf{N}^{\mathbf{0}} \text { atendimentos } \\
\text { Mês } \mathbf{1}\end{array}$ & $\begin{array}{c}\mathbf{N}^{\mathbf{0}} \text { atendimentos } \\
\text { Mês } \mathbf{2}\end{array}$ & Total & \% \\
\hline Dor em abdome & 236 & 254 & 490 & 15,9 \\
\hline Mal estar em adulto & 188 & 230 & 418 & 13,6 \\
\hline Cefaleia & 137 & 148 & 285 & 7,3 \\
\hline Dor lombar & 111 & 124 & 235 & 7,5 \\
\hline Dor torácica & 108 & 122 & 230 & 5,9 \\
\hline Dor de garganta & 76 & 104 & 180 & 5,6 \\
\hline Dispneia & 85 & 88 & 173 & 4,9 \\
\hline Problemas em & 64 & 86 & 150 & 4,5 \\
\hline extremidades & 67 & 72 & 139 & 4,1 \\
\hline Erupção cutânea & 57 & 70 & 127 & 3,2 \\
\hline Problemas urinários & 47 & 52 & 99 & 2,8 \\
\hline Vertigem e tonturas & 48 & 40 & 88 & 2,8 \\
\hline Diarreia & 37 & 49 & 86 & 2 \\
\hline Vômitos & 26 & 36 & 62 & 10,3 \\
\hline Mordeduras e & 152 & 316 & 100 \\
\hline picadas & 164 & 1627 & 3078 & \\
\hline Outras queixas & 1451 & & & \\
\hline Total & & &
\end{tabular}

Do total de registros com classificação de risco (3.078), 1.031 (33,49\%) disseram ter buscado outro serviço de saúde antes do HU, retornando na ocasião a esta instituição por não terem sido atendidos (ausência de médico, sem vaga para consulta de emergência); terem sido atendidos de forma insatisfatória (sem melhora com tratamento realizado, fragilidade de recursos para apoio diagnóstico - ausência de laboratório, RX danificado); terem sido encaminhados (verbalmente/ carta de encaminhamento); entre outras justificativas. Já 1.965 (63,84\%) relataram ter vindo direto ao SEA/HU devido à proximidade (trabalham nos arredores, estudantes/servidores públicos, moradores da região, turista), à preferência (serviço de melhor qualidade), por já fazer tratamento no HU, por ser referência, ou ainda por ser hospital escola e contar com profissionais mais dispostos a realizar a investigação diagnóstica.

Com relação à procedência dos usuários do SEA/HU, identificou-se que 2.016 usuários $(65,50 \%)$ eram provenientes da localidade onde se situa este serviço, levando a crer que a procura pode estar relacionada com a proximidade geográfica do serviço. Todos eles são procedentes do município-sede, distribuídos segundo o distrito sanitário. Os demais usuários vieram de municípios vizinhos $(20,8 \%)$ ou outros municípios do próprio estado e de outros estados do Brasil (13,10\%), ou em turismo (0,6\%) (Tabela 3). 
Tabela 1 - Distribuição de usuários classificados de acordo com protocolo de Manchester adaptado. SEA/ HU. Florianópolis-SC-Brasil, 2012

\begin{tabular}{lcc}
\hline Procedência & $\mathbf{N}^{\mathbf{0}}$ de usuários & $\mathbf{\%}$ \\
\hline Distrito Sanitário Sul & 514 & 16,7 \\
\hline Distrito Sanitário Norte & 385 & 12,5 \\
\hline Distrito Sanitário Continente & 159 & 5,17 \\
\hline Distrito Sanitário Leste & 416 & 13,5 \\
\hline Distrito Sanitário Centro & 542 & 17,6 \\
\hline Subtotal Município-sede & $\mathbf{2 . 0 1 6}$ & $\mathbf{6 5 , 5}$ \\
\hline Municípios vizinhos & 640 & 20,8 \\
\hline Outros municípios & 403 & 13,1 \\
(dentro e fora do estado) & 19 & 0,6 \\
\hline Outros locais (turismo) & $\mathbf{1 . 0 6 2}$ & $\mathbf{3 4 , 5}$ \\
\hline Subtotal demais localidades & 3.078 & 100 \\
\hline Total & &
\end{tabular}

Com base nos dados coletados durante a observação participante, foi possível identificar alguns aspectos que vêm contribuindo para o excesso da demanda no SEA/HU.

Uma observação importante diz respeito aos usuários classificados com as cores verde e azul. Estes permanecem um tempo longo (tempo maior do que o previsto pelo protocolo), geralmente horas, para receberem atendimento e, muitas vezes, não suportam a espera e desistem do atendimento ou, ainda, se revoltam contra a equipe de profissionais.

O número insuficiente de profissionais médicos para atender a demanda do serviço constituiu-se, muitas vezes, um reflexo desta demora, somando-se ao fato que dependendo da gravidade dos pacientes e das necessidades de cuidado não havia recursos humanos suficientes, tão pouco estrutura física para atender a todos. Durante os meses do desenvolvimento deste estudo, a mídia esteve presente no SEA/HUH, divulgando a questão da superlotação no serviço.

A dificuldade de acesso aos demais serviços de saúde e a ineficiência do atendimento é relatada pelos usuários, tanto ao procurarem as Unidades de Pronto Atendimento (UPA) como a Atenção Primária à Saúde (APS), sendo esta última a de maior incidência. A justificativa diz respeito à ausência de melhora no quadro clínico. Já a justificativa relacionada exclusivamente à APS seria a falta de profissionais médicos para realizar as consultas e um horário limitado para as consultas de "emergência".

A questão cultural observada como agravante para excesso de demanda ao SEA/HU diz respeito a aspectos de preferência, indicação de familiar, qualidade do serviço, proximidade geográfica, referência para casos de intoxicação.

\section{DISCUSSÃO}

Durante as últimas décadas, estudos ${ }^{(6-7)}$ revelaram um aumento na utilização incorreta dos SEH (65\% dos problemas poderia ser resolvido em serviços ambulatoriais), demanda esta que extrapola os recursos oferecidos e ocasiona longas esperas por atendimento, corroborando aos dados encontrados no SEA/HU. O fato de que $63,55 \%$ dos usuários busca o SEH para solucionar casos que deveriam ser resolvidos em outro nível da RAS do SUS demonstra uma incipiente e frágil estruturação da RUE. Estudos(4-6) mostram que os usuários não diferenciam situações emergenciais de acordo com o conceito técnico, nem compreendem quais os serviços que compõem a RUE. Assim, o SEH funciona, ainda, como uma principal porta de entrada para o sistema de saúde.

A demanda de usuários com queixas classificadas como não urgentes (verde e azul) pode ser encaminhada por profissional capacitado a outros serviços da RUE, desde que esteja garantido o acesso. Estudo realizado em hospital público ${ }^{(12)}$ demonstrou que parcela importante $(36 \%)$ de usuários, acolhidos com classificação de risco por enfermeiros, foram encaminhados a outros serviços, resultando uma 
redução significativa dos atendimentos médicos. Neste sentido, procedeu-se tratamento imediato a pessoas que efetivamente dele necessitavam.

Com relação ao quantitativo de profissionais para atender a demanda do serviço emergencial, estudo realizado no oeste do Paraná destaca que, além de insuficiência no quantitativo, há situações cujas intervenções ultrapassam o âmbito multiprofissional e incluem outras esferas administrativas, inerentes às decisões de governo $^{(13)}$. Nesta mesma perspectiva, o modo de organização do processo de trabalho da APS, com incipiente capacidade de resposta e tempo limitado para atender a demanda emergencial, provoca o comprometimento da porta de entrada hospitalar.

Apesar dos avanços ocorridos nas políticas de saúde com implementação de diretrizes de humanização e acolhimento ao usuário em todos os níveis do sistema ${ }^{(1-2,9)}$, permanece na cultura popular a ideia da busca por resolutividade em serviços de pronto atendimento ou de emergência hospitalar. Estudos ${ }^{(13-14)}$ mostram que os serviços que privilegiam o agendamento à demanda espontânea faz com que usuários sejam encaminhados informalmente a outros serviços, quando a demanda excede ao programado ou não corresponde ao que é ofertado pelo serviço.

Ambas as situações fazem com que o usuário recorra aos SEH mesmo para os casos que também acreditam poder ser resolvidos nos demais pontos de atenção. A população consegue perceber maior agilidade ou qualidade em situações de urgência e emergência pelo SEH e por isso procuram este serviço, bem como, notam que para problemas solucionáveis em outros níveis de atenção a demora é maior. No entanto, relatam dificuldade de mudar esta prática por razões diversas, entre elas pode-se citar a localização do serviço, a maior qualidade ou agilidade na assistência prestada e as experiências positivas vivenciadas e/ou relatadas ${ }^{(14)}$.

O excesso de demanda pode estar associada à questão cultural, também reconhecida por outros autores $^{(15)}$, com características similares de procura pelo serviço de emergência: por sugestão de familiar; crença como melhor condição solução do problema; facilidade de exames no momento da consulta.

O fato de se tratar de um hospital público, bem como a inexistência de UPA em três dos cinco distritos de saúde, pode explicar a procura pelo SEA/HU pela população local. Contudo, em dois distritos que possuem UPA, pode ser sugestivo de falta de apoio diagnóstico ou de insuficiência de pessoal/material para atender a demanda ${ }^{(1-2,8)}$.

Apesar do SEA/HU contar com o sistema de classificação de risco implantado, e este ser considerado uma estratégia potencialmente decisiva na reorganização e implementação da promoção de saúde em rede ${ }^{(8)}$, é necessário ir além. Com esta perspectiva, as técnicas de coleta e análise de dados possibilitaram tecer alguns indicativos a respeito da organização da porta de entrada do SEH e, também, em relação aos demais pontos de atenção da RAS/RUE.

Um primeiro indicativo diz respeito à criação, em parceria entre equipe do SEA/HU e a Secretaria Municipal de Saúde e suas unidades locais, de protocolos de encaminhamento para usuários classificados como verde (pouco urgente), azul (não urgente) e branco (procedimento). De acordo com as diretrizes da RUE, é necessário articulação e integração dos diversos serviços e equipamentos de saúde para que se possa constituir redes de saúde com conectividade entre os pontos de atenção ${ }^{(1-2)}$. O estabelecimento de parcerias entre os profissionais que integram RAS/RUE poderá induzir o esclarecimento ao usuário para o acesso do serviço de saúde preferencialmente a partir da APS. Além disso, as parcerias motivadas para a continuidade da assistência refletem as ações direcionadas ao cumprimento dos princípios do SUS.

A sensibilização da equipe que trabalha no SEA/HU, por meio de técnicas de integração que explorem o conhecimento de cada ator frente à RAS/RUE, constitui-se condição diferenciada para organização do serviço. O trabalho em equipe e as relações interprofissionais no contexto das práticas de atenção à saúde estão, ainda, no plano do anseio. É necessário, portanto, fomentar o protagonismo dos sujeitos envolvidos no cuidado, numa perspectiva de gestão que os tornem corresponsáveis pela atenção prestada ${ }^{(16)}$. Paralelamente, a instituição de protocolos assistenciais para realização de procedimentos já prescritos, negociados entre os integrantes da equipe, permite sistematizar adequadamente a assistência. Os benefícios resultantes correspondem a uma melhor qualidade do serviço prestado e, também, em segurança 
para o profissional que desenvolve a ação ${ }^{(17)}$.

Embora não tenha sido sistematizado em dados, observou-se uma demanda maior ao SEA/ HU nas segundas-feiras, oposta à observada nos finais de semana. Este é um importante ponto de reflexão, visto que a sobrecarga de trabalho pode ocorrer pelo volume da demanda em determinados períodos. Estudos que demonstrem a pertinência de redistribuição dos turnos de trabalho, com acréscimo de profissionais nos turnos/dia de maior demanda, são necessários não só como tecnologia de gestão de pessoas em ambiente hospitalar como também em qualidade da assistência e redução de sobrecarga da equipe. Por outro lado, são complexas as questões que circundam a relação entre os recursos humanos e a qualidade dos serviços de saúde ${ }^{(18)}$. Em geral, há insatisfação profissional gerada pelo excesso de trabalho e, muitas vezes, despreparo no atendimento dos casos nos serviços de emergência, o que requer uma ação governamental para a suficiência e enfrentamento de fragilidades de vínculos profissionais ${ }^{(18)}$.

Com relação aos demais pontos de atenção da RAS/RUE, a identificação de fragilidades tanto na APS quanto nas UPAs, no atendimento às urgências, podem subsidiar intervenções operativas neste campo de atuação. Os serviços de emergência têm se tornado uma válvula de escape dos serviços de saúde ${ }^{(19)}$ e, neste sentido, a ampliação do acesso na APS para realização de acolhimento dos casos agudos de menor complexidade já se constitui uma propulsão de mudança na RUE ${ }^{(1-2)}$. Em outras palavras, $\mathrm{O}$ acolhimento dos casos agudos, com classificação de risco, em todos os pontos de atenção da RUE, definirá melhor as práticas e intervenções adequadas aos diferentes agravos que demandam a estes serviços. Para tanto, reforça-se a necessária capacitação de todos os profissionais da RUE para o primeiro atendimento ao usuário do SUS ${ }^{(2)}$.

\section{CONSIDERAÇÕES FINAIS}

Este estudo demonstrou que a demanda do SEA/HU é alta, porém não corresponde à sua atribuição específica. A maioria dos usuários recorre a este serviço para solucionar casos não emergenciais. Isso pode ser justificado por diferentes motivos, dentre os quais o não reconhecimento do fluxo da RAS/RUE; a incipiente difusão de informações a respeito destas redes; a comodidade do usuário em ser atendido de forma imediata, por se tratar de serviço mais próximo da sua casa, trabalho ou escola; a característica do atendimento à demanda emergencial; a recente implementação do acolhimento de casos agudos nos demais pontos de atenção, em especial, na APS e UPA; a necessária capacitação de profissionais.

A resolução e organização da porta de entrada da RUE, com acesso aos demais serviços de saúde e suficiência de apoio diagnóstico, podem contribuir para mudar o perfil da SEA/ HU e, generalizando, do SEH. A comunicação entre os pontos de atenção constitui fator decisivo para esclarecimento dos cidadãos, especialmente em relação ao cuidado continuado e acompanhamento na rede básica de saúde, com diagnósticos e tratamentos adequados.

A integralidade dos serviços, levando em conta a qualificação do sistema de referência e contrarreferência, contribui para o processo de orientar os usuários na busca do serviço indicado para a complexidade do seu problema de saúde. Contudo, novos estudos com esta perspectiva, com coleta mais ampla de dados, poderão não só corroborar os resultados aqui encontrados ou apresentar outros aspectos não evidenciados neste estudo, mas ampliar indicativos de novas tecnologias de organização do cuidado em rede.

\section{REFERÊNCIAS}

1. Brasil. Decreto n. 7.508, de 28 de junho de 2011. Regulamenta a Lei n. 8.080, de 19 de setembro de 1990 para dispor sobre a organização do Sistema Único de Saúde - SUS, o planejamento da saúde, a assistência à saúde e a articulação interfederativa, e dá outras providências [Internet] [acesso em 10 nov 2011]. Disponível: http://www.jusbrasil.com.br/ legislacao/1028206/decreto-7508-11

2. Ministério da Saúde (BR). Portaria GM n. 1.600, de 07 de julho de 2011. Reformula a Política Nacional de Atenção às Urgências e institui a Rede de Atenção às Urgências no Sistema Único de Saúde (SUS). Diário Oficial da União, Brasília, DF, 12 jul. 2011.

3. Bittencourt J, Hortale VA. Intervenções para solucionar a superlotação nos serviços de emergência hospitalar: uma revisão sistemática. Cad. Saúde Pública. [Internet] 2009;25(7) [acesso em 28 set 2011]. Disponível: http:// dx.doi.org/10.1590/S0102-311X2009000700002

4. Valentim MRS, Santos MLSC. Políticas de saúde em 
emergência e a enfermagem. Rev. enferm. UERJ. 2009;17(2):285-9.

5. Rossaneis MA, Haddad MCL, Borsato FG, Vannuchi MO, Sentone ADD. Caracterização do atendimento após implantação do acolhimento, avaliação e classificação de risco em hospital público. Rev. Eletr. Enf. [Internet] 2011;13(4) [acesso em 20 out 2013]. Disponível: http://www.revistas.ufg.br/index.php/fen/ article/view/10182/10195

6. Nascimento ERP, Hilsendeger BR, Neth C, Belaver GM, Bertoncello KCG. Classificação de risco na emergência: avaliação da equipe de enfermagem. Rev. enferm. UERJ. 2011;19(1):84-8.

7. O'dwyer GO, Oliveira SP, Seta MH. Avaliação dos serviços hospitalares de emergência do programa QualiSUS. Ciênc. saúde colet. 2009;14(5):1881-90.

8. Mendes EV. As redes de atenção à saúde. Brasília: Organização Pan-Americana da Saúde; 2011.

9. Ministério da Saúde (BR). Secretaria de Atenção à Saúde. Política Nacional de Humanização da Atenção e Gestão do SUS. Acolhimento e classificação de risco nos serviços de urgência. Brasília: Ministério da Saúde; 2009.

10. Minayo MCS. O desafio do conhecimento: Pesquisa qualitativa em saúde. 11ª ed. São Paulo: Hucitec; 2008.

11. Hospital Universitário Polydoro Ernani de São Thiago. Florianópolis (SC): Universidade Federal De Santa Catarina, 2014. [Internet] [acesso em 21 out 2013]. Disponível: http://www.hu.ufsc.br/portal_novo/?page_ $\mathrm{id}=12$

12. Rossaneis MA, Haddad MCL, Borsato FG, Vannuchi $\mathrm{MO}$, Sentone ADD. Caracterização do atendimento após implantação do acolhimento, avaliação e classificação de risco em hospital público. Rev. Eletr. Enf. [Internet] 2011;13(4) [acesso em 22 set 2012]. Disponível: http://www.fen.ufg.br/fen_revista/v13/n4/ pdf/v13n4a08.pdf

13. Maciak I, Sandri JVA, Spier FD. Humanização da assistência de enfermagem em uma unidade de emergência: percepção do usuário. Cogitare enferm. 2009;14(1) [acesso em 28 nov 2012]. Disponível: http://ojs.c3sl.ufpr.br/ojs/index.php/cogitare/article/ viewFile/14269/9592

14. Marques GQ, Lima MADS. Demandas de usuários a um serviço de pronto atendimento e seu acolhimento ao sistema de saúde. Rev. Latino-Am. Enfermagem. [Internet] 2007;14(1) [acesso em 28 nov 2012]. Disponível: http://dx.doi.org/10.1590/S010411692007000100003

15. Carret MLV, Fassa AG, Paniz VMV, Soares PC. Características da demanda do serviço desaúde de emergência no Sul do Brasil. Ciênc. saúde colet. 2011;16(suppl 1):1069-79.

16. Cardoso CG, Hennington EA. Trabalho em equipe e reuniões multiprofissionais de saúde: uma construção à espera pelos sujeitos da mudança. Trab. educ. saúde [Internet]. 2011;9 (suppl 1) [acesso em 7 nov 2012] Disponível: http://dx.doi.org/10.1590/S198177462011000400005

17. Ministério da Saúde (BR). Departamento de Ciência e Tecnologia. Coordenação Geral de Avaliação de Tecnologias em Saúde. Avaliação de Tecnologias em Saúde no Brasil, 2014 [Internet] [acesso em 10 nov 2012]. Disponível: http://portal.saude. gov.br/portal/saude/profissional/visualizar_texto. cfm?idtxt=29084\&janela=2

18. Dubeux LS, Freese E, Reis YAC. Avaliação dos serviços de urgência e emergência da rede hospitalar de referência no nordeste brasileiro. Cad. Saúde Pública. 2010;26(8):1508-18.

19. Júnior JLCA, Furtado BMASM, Cavalcanti P. O perfil da emergência do hospital da restauração: uma análise dos possíveis impactos após a municipalização dos serviços de saúde. Rev. Bras. Epidemiol. 2004;7(3):27989. 